\title{
Assessment of Genotype $\times$ Environment Interaction and Stability Analysis in Indian Mustard (Brassica juncea L.)
}

\author{
Neelam Shekhawat ${ }^{1 *}$, Hemlata Sharma ${ }^{2}$ and Bheeru Lal Meena ${ }^{3}$ \\ ${ }^{1}$ ICAR-National Bureau of Plant Genetic Resources, Regional Station, \\ Jodhpur-342005, Rajasthan, India \\ ${ }^{2}$ Rajasthan College of Agriculture, Maharana Pratap University of Agriculture and \\ Technology, Udaipur- 313001, Rajasthan, India. \\ ${ }^{3}$ ICAR-Directorate of Rapeseed-Mustard Research, \\ Sewar, Bharatpur 321303 (Rajasthan), India \\ *Corresponding author
}

\section{A B S T R A C T}

\section{Keywords}

Indian mustard, Stability, Seed yield, Regression, $\mathrm{G} \times \mathrm{E}$ interaction

Article Info

Accepted:

15 March 2020 Available Online: 10 April 2020

\begin{abstract}
Fifty-five Indian mustard genotypes comprising forty-five hybrids and ten parental genotypes along with a two checks viz., NRCHB-506 and DMH-1 were evaluated for stability parameters of morphological and biochemical characters over the three different environments. Pooled analysis of variance over three environments showed that the genotypic variance when tested against $\mathrm{G} \times \mathrm{E}$ were significant for all the eight characters when tested against pooled error.Pooled deviation effect was significant for all the traits except protein content. The parents, parents Pusa Vijay and Bio-902 showed their suitability for unfavourable environment for 1000 seed weight.Two crosses Pusa Vijay $\times$ Bio-902 and Pusa Vijay $\times$ RB-50 had suitability for unfavourable environment while cross Pusa Vijay $\times$ RB-50 exhibited its suitability for favourable environment for seed yield per plant. For protein content, only one cross Pusa Vijay $\times$ PM-30showed its suitability for unfavourable environment, thereby this cross would have specific adaptability to poor yielding environments. These genotypes can be recommended for varied environments to exploit their high yield potential with morphological traits.
\end{abstract}

\section{Introduction}

Indian mustard [Brassica juncea $(\mathrm{L})$ Czern \& Coss] is an important oilseed crop of Brassicaceae family which accounts more than $70 \%$ under rapeseed and mustard. The Brassicaceae, contains about 3500 species and
350 genera, is one of the 10 most economically important plant families. Rapeseed-mustard comprising eight different species viz. Indian mustard, toria, yellow sarson, brown sarson, gobhisarson, karan rai, black mustard and taramira are being cultivated in 53 countries spreading all over 
the world. In India, mustard and rape seed are being grown largely in states like Uttar Pradesh, Rajasthan, Haryana, Assam, Gujarat, Punjab, West Bengal and Madhya Pradesh. Mustard seed is largely crushed for oil, which is perhaps the cheapest source of oil in our daily diet. Mustard seeds contain about 38$42 \%$ oil, which is golden yellow, fragrant and considered among the healthiest and most nutritional cooking medium. In addition to this, it is also utilized as condiment, for medicinal uses and in preparation of soaps, hair oil, lubricants, paints, plasticizers and as a condiment in pickles.

Stability in performance of genotype for economic characters is one of the desirable properties for selection or recommendation of new genotypes in plant breeding program. For this purpose, growing of breeding lines over time and space has become an integral part of any crop improvement programme. Despite such vigorous evaluation and subsequent selection, genotypes $\mathrm{x}$ environments interaction have been a major problem for consistent performance of selected genotypes. It is commonly observed that many varieties of different crops do not exhibit consistent performance, when tested under different environments. Such interaction, result in change of relative ranking of different genotypes and also alters magnitude of difference between genotypes, which create problem for plant breeders in making proper assessment of genotypes, when the same are tested over varied environments. The progress resulting from selection is also reduced due to effect of large genotypes $x$ environments interaction. It may be noted that genotypes $\mathrm{x}$ environments interaction exists regardless of genetic material with which a breeder is working.

A knowledge regarding nature and relative magnitude of genotypes $\mathrm{x}$ environments interaction is important in making decisions concerning breeding methods, selection programme and testing procedures in crop plants (Baker, 1969). In order to minimize genotypes $\mathrm{x}$ environments interactions, and to increase precision in selection, stratification of environments has been employed; however, even with refinement of technique, an interaction of genotypes with environments within same year remains very large (Bradshaw, 1965). Sprague (1966) opined that the possibility of reducing genotypes $x$ environments $(\mathrm{G} \quad \mathrm{x}$ E) interaction in field experiments is questionable despite knowledge of the factors responsible for such interactions.

\section{Materials and Methods}

Fifty-five Indian mustard genotypes comprising forty-five crosses and ten parental genotypes along with a two checks viz., NRCHB-506 and DMH-1 were evaluated in randomized block design with three replications during Rabi 2015-2016 at three locations viz., Instructional Farm RCA, Udaipur $\left(\mathrm{E}_{1}\right)$, Instructional Farm CTAE, Udaipur $\left(\mathrm{E}_{2}\right)$ and Instructional Farm, KVK Chittorgarh $\left(E_{3}\right)$. A single row plot of four meter length was planted with crop geometry of $30 \mathrm{~cm} \mathrm{x} 10 \mathrm{~cm}$. The recommended agronomical practices were adopted to raise a healthy crop.

Observations were recorded on five randomly selected competitive plants in each genotype in each replication for various characters viz., days to $50 \%$ flowering, days to maturity, plant height, number of primary branches per plant, number of secondary branches per plant, length of main branch, number of silique per plant, length of silique, number of seeds per plant, 1000 seed weight, seed yield per plant, harvest index, biological yield per plant, oil content and protein content. Mean data obtained was statistically analysed and the genotypes were assessed for their stability of 
performance across environments following the method described by Eberhart and Russell (1966).

\section{Results and Discussion}

Plant breeder attempts to produce progressively better-adapted populations to the existing or altered environments. Stability parameters have proved to be valuable for assessing the response of various genotypes under changing environmental conditions.

The quantitative characters are highly influenced by the environment. The magnitude of this influence is reflected by $G$ $\times \mathrm{E}$ interaction. This interaction generally remains confounded with variance and leads to biased estimates of genetic parameters. This bias can be minimized by growing the breeding materials over the environments. The study of $\mathrm{G} \times \mathrm{E}$ interactions was made as per the procedure outlined by Eberhart and Russell (1966).

\section{Analysis of Variance}

Pooled analysis of variance over three environments showed that the genotypic variance when tested against $G \times E$ were significant for all the eight characters (Table 1). Partitioning of $G \times E$ interaction showed that Environment linear interaction effects were non-significant for all the eight traits and $(\mathrm{G} \times \mathrm{E})$ linear interactions were highly significant for all the traits. Pooled deviation effect was significant for all the traits except protein content. Further, partitioning of variance i.e. Environment + (Genotypex Environment) interaction was observed to be significant all the traits. The Magnitude of mean square due to Environment + (Genotype $\times$ Environment) was also recorded higher as compared to Genotype $\times$ Environment (linear) for all the characters except protein content.

\section{Stability parameter}

Since, bartlet test was detected nonsignificant for days to $50 \%$ flowering, plant height, number of primary branches per plant, length of main branch, seed yield per plant, 1000 seed weight, harvest index and protein content and the stability parameters in respect of these eight traits were compared to evaluate relative stability of fifty-seven different genotypes (ten parents, forty-five crosses and two checks) as per Eberhart and Russell' 1966. The results are presented in Table 2 to 5 .

The genotypes with higher mean values, regression coefficient value of unity $(b i=1)$ and non-significant deviations from linear regression deviation $\left(\overline{\mathrm{S}}^{2} \mathrm{di}=0\right)$ were considered as stable for the traits and adaptable to varied environmental conditions studied in the present investigation. However, genotypes with a higher mean value with nonsignificant deviations from linear regression and regression coefficient near to unity were considered to be responsive and suitable for favorable environmental conditions.

Further, the genotypes with higher mean values and regression coefficient less than one (unity) or negative and non-significant deviations from linear regression were considered to be responsive and suitable for poor environmental conditions. Accordingly, the genotypes were classified into different classes as suitable for varied environmental condition.

For the trait days to $50 \%$ flowering, none of the parent showed significant regression coefficient while among the crosses, one cross RB-50 × RGN-48 exhibited non-significant deviation from regression, lower mean (50.44) than mean of crosses (50.12 days) with regression coefficient less than unity. 
Thus, this cross showed its suitability for favorable environment. For plant height, two crosses viz., Bio-902 $\times$ DRMR-IJ-31 and RGN-229 $\times$ RGN-48showed non-significant deviation from regression with mean value (189.11 and $186.78 \mathrm{~cm}$, respectively), higher than the mean of crosses $(181.98 \mathrm{~cm})$. Out of these, one cross Bio-902 $\times$ DRMR-IJ-31 exhibited regression coefficient more than unity with mean plant height of $189.11 \mathrm{~cm}$ expressed its suitability for favorable environment. While another cross RGN-229 $\times$ RGN-48 expressed higher mean $(186.78 \mathrm{~cm})$ and regression coefficient less than unity, therefore expressed its suitability for unfavorable environment.

Parent Bio-902 exhibited higher mean (7.53) than parental mean (6.41). Likewise, cross Pusa Vijay $\times$ RGN-229 also showed higher mean (6.16) than mean of the crosses (6.10). Parent Bio-902 and cross Pusa Vijay $\times$ RGN229 both exhibited non-significant deviation from regression, regression coefficient about unity, showed their suitability for average environment.For the trait length of main branch, only one cross Bio-902 $\times$ Laxmi exhibited higher mean (6.76) than mean of crosses (6.58) with non-significant deviation from regression and regression coefficient more than one. Thus, this cross exhibited its suitability for favourable environment.

Two crosses Pusa Vijay $\times$ Bio-902 and Pusa Vijay $\times$ RB-50 showed higher mean than the parental mean $(31.84 \mathrm{~g})$ for seed yield per plant. Both these crosses exhibited nonsignificant deviation from regression. Cross Pusa Vijay $\times$ Bio-902 depicted higher mean $(39.99 \mathrm{~g})$ regression coefficient less than unity thereby showed its suitability for unfavourable environment.

Further, cross Pusa Vijay $\times$ RB-50 exhibited higher mean $(34.10 \mathrm{~g})$ with regression coefficient more than unity which expressed its suitability for favourable environment. Among parents, two parents Pusa Vijay and Bio-902 showed their mean for 1000 seed weight higher than parental mean $(5.06 \mathrm{~g})$. Both the parents showed non-significant deviation from regression, regression coefficient less than unity and showed their suitability for unfavorable environment.

Table.1 Analysis of variance Eberhart and Russel (1966)

\begin{tabular}{|c|c|c|c|c|c|c|c|}
\hline SN & Characters & Genotype & $\mathbf{E}+(\mathbf{G} \times \mathbf{E})$ & $\mathbf{E}(\mathbf{L})$ & $G \times E(L)$ & Pool dev. & Pool Err \\
\hline & & [56] & [114] & [1] & [56] & [57] & [336] \\
\hline 1 & Days to $50 \%$ flowering & $44.6870 * *$ & $4.8623 * *$ & 0.0017 & $3.7530 * *$ & $6.0374 * *$ & 0.9741 \\
\hline 2 & Plant height $(\mathrm{cm})$ & $149.2952 * *$ & $36.2346 * *$ & 0.0668 & $36.4870^{*}$ & $36.6211 *$ & 24.98 \\
\hline 3 & $\begin{array}{c}\text { No. of primary branches } \\
\text { Per plant }\end{array}$ & $0.6143 * *$ & $0.5437 * *$ & 0.0034 & $0.5331 * *$ & $0.5636 * *$ & 0.1364 \\
\hline 4 & $\begin{array}{l}\text { Length of main branch } \\
\qquad(\mathrm{cm})\end{array}$ & $86.1009 * *$ & $25.5599 * *$ & 0.0336 & $27.1162 * *$ & $24.4787 * *$ & 4.532 \\
\hline 5 & Seed yield per plant $(\mathrm{g})$ & $64.5820 * *$ & $9.3606^{* *}$ & 0.0029 & $6.2233^{* *}$ & $12.6071 * *$ & 2.895 \\
\hline 6 & 1000 seed weight(g.) & $0.6494 * *$ & $0.1747 * *$ & 0.0001 & $0.1260 * *$ & $0.2256 * *$ & 0.03501 \\
\hline 7 & Harvest index & $60.3987 * *$ & $43.4796 * *$ & 0.0436 & $34.2310^{* *}$ & $53.3278 * *$ & 7.256 \\
\hline 8 & Protein content $(\%)$ & $7.5084 * *$ & $0.6339 * *$ & 0.0005 & $0.7651 * *$ & 0.5161 & 0.3948 \\
\hline
\end{tabular}


Table.2 Stability parameters for days to 50\%flowering and plant height

[Eberhart and Russel (1966)]

\begin{tabular}{|c|c|c|c|c|c|c|c|}
\hline \multirow[t]{2}{*}{ SN } & \multirow[t]{2}{*}{ Genotype } & \multicolumn{3}{|c|}{ Days to $50 \%$ flowering } & \multicolumn{3}{|c|}{ Plant height } \\
\hline & & $\mu$ & bi & $\mathbf{s}^{2} d_{i}$ & $\mu$ & bi & $\mathbf{s}^{2} d_{i}$ \\
\hline 1 & $\mathrm{P} 1$ & 55.67 & 4.76 & 1.078 & 181.11 & 3.83 & $75.704 *$ \\
\hline 2 & $\mathrm{P} 2$ & 49.22 & 4.93 & $5.877 * *$ & 183.33 & -0.82 & -14.886 \\
\hline 3 & P3 & 54.67 & -0.94 & -0.391 & 181.00 & -2.70 & -23.224 \\
\hline 4 & $\mathrm{P} 4$ & 44.22 & 5.38 & -0.121 & 201.22 & 1.69 & -24.893 \\
\hline 5 & P5 & 50.11 & -16.24 & 0.699 & 197.22 & -0.04 & -21.798 \\
\hline 6 & P6 & 52.89 & 18.19 & $4.925 *$ & 182.78 & 3.35 & 1.352 \\
\hline 7 & $\mathrm{P} 7$ & 56.11 & 2.18 & 2.199 & 182.78 & -2.13 & $151.524 * *$ \\
\hline 8 & $\mathrm{P} 8$ & 47.78 & -3.27 & $2.965^{*}$ & 180.44 & 4.17 & -24.980 \\
\hline 9 & P9 & 47.22 & -1.57 & $21.085^{* *}$ & 174.56 & 3.79 & -6.378 \\
\hline 10 & P10 & 54.44 & 3.65 & 0.048 & 190.22 & 2.44 & $132.800 *$ \\
\hline 11 & P1 x P2 & 52.67 & 0.49 & -0.108 & 181.78 & $2.11 *$ & -24.914 \\
\hline 12 & P1 x P3 & 54.22 & -4.79 & 2.682 & 176.56 & 1.33 & -9.215 \\
\hline 13 & P1 x P4 & 49.56 & 2.33 & $6.579 * *$ & 198.22 & 0.40 & $76.704 *$ \\
\hline 14 & P1 x P5 & 51.33 & 0.49 & -0.108 & 188.89 & 1.39 & 11.286 \\
\hline 15 & P1 x P6 & 54.67 & 0.73 & 0.975 & 177.67 & -0.85 & 7.180 \\
\hline 16 & P1 x P7 & 55.56 & 0.58 & $8.845 * *$ & 178.67 & 0.38 & 20.684 \\
\hline 17 & P1 x P8 & 53.33 & 0.69 & 0.536 & 175.33 & 4.00 & -11.840 \\
\hline 18 & P1 x P9 & 52.44 & -1.52 & $14.212 * *$ & 173.33 & -3.25 & -24.515 \\
\hline 19 & $\mathrm{P} 1 \times \mathrm{P} 10$ & 55.67 & 5.69 & -0.526 & 184.67 & 5.09 & $149.261 * *$ \\
\hline 20 & $\mathrm{P} 2 \times \mathrm{P} 3$ & 52.78 & 5.31 & 1.726 & 179.56 & 0.13 & -17.860 \\
\hline 21 & $\mathrm{P} 2 \times \mathrm{P} 4$ & 41.44 & -2.01 & $7.825 * *$ & 178.89 & 3.76 & $192.698 * *$ \\
\hline 22 & P2 x P5 & 51.33 & -12.28 & $3.229 *$ & 190.56 & 4.09 & -2.911 \\
\hline 23 & P2 x P6 & 51.22 & 6.56 & -0.582 & 177.67 & 2.70 & -23.227 \\
\hline 24 & $\mathrm{P} 2 \times \mathrm{P} 7$ & 53.56 & 1.60 & 0.855 & 179.67 & $-0.71+$ & -24.921 \\
\hline 25 & $\mathrm{P} 2 \times \mathrm{P} 8$ & 47.78 & 6.94 & -0.852 & 175.89 & 3.26 & -24.283 \\
\hline 26 & $\mathrm{P} 2 \times \mathrm{P} 9$ & 48.33 & -8.30 & -0.685 & 174.67 & -4.74 & -24.185 \\
\hline 27 & $\mathrm{P} 2 \times \mathrm{P} 10$ & 51.11 & 8.16 & $2.938^{*}$ & 186.44 & -1.76 & 35.472 \\
\hline 28 & $\mathrm{P} 3 \times \mathrm{P} 4$ & 44.11 & 0.11 & 2.654 & 179.67 & 1.01 & 17.997 \\
\hline 29 & P3 x P5 & 49.78 & 5.31 & 1.726 & 185.00 & $1.11 *$ & -24.977 \\
\hline 30 & P3 x P6 & 53.78 & 0.60 & $7.066 * *$ & 180.22 & -2.60 & -23.655 \\
\hline 31 & P3 x P7 & 53.78 & 5.97 & $3.912 *$ & 172.44 & 3.35 & 1.354 \\
\hline 32 & P3 x P8 & 49.56 & -1.29 & 1.607 & 182.22 & 2.07 & 24.730 \\
\hline 33 & P3 x P9 & 49.44 & 0.03 & $10.211 * *$ & 174.44 & -0.71 & -18.619 \\
\hline 34 & $\mathrm{P} 3 \times \mathrm{P} 10$ & 52.89 & -6.70 & -0.312 & 169.67 & $3.56 * *++$ & -24.979 \\
\hline 35 & P4 x P5 & 40.78 & 4.13 & $2.795 *$ & 199.44 & 2.63 & -13.450 \\
\hline 36 & P4 x P6 & 44.78 & 5.07 & 0.414 & 199.33 & 1.27 & -24.268 \\
\hline 37 & P4 x P7 & 49.22 & 0.58 & $8.845 * *$ & 185.33 & -0.13 & 4.510 \\
\hline 38 & P4 x P8 & 44.22 & 6.81 & 0.216 & 189.11 & $3.21 *+$ & -24.891 \\
\hline
\end{tabular}




\begin{tabular}{|l|l|r|r|r|r|r|r|}
\hline $\mathbf{3 9}$ & P4 x P9 & 43.89 & -6.01 & -0.803 & 179.56 & 3.44 & -23.299 \\
\hline $\mathbf{4 0}$ & P4 x P10 & 47.22 & 6.36 & $6.781 * *$ & 188.89 & 5.75 & 4.002 \\
\hline $\mathbf{4 1}$ & P5 x P6 & 51.67 & 12.85 & $16.081 * *$ & 186.56 & -1.39 & 52.185 \\
\hline $\mathbf{4 2}$ & P5 x P7 & 51.89 & -4.54 & $4.678^{*}$ & 183.44 & 6.11 & -5.583 \\
\hline $\mathbf{4 3}$ & P5 x P8 & 46.00 & -3.13 & $10.752^{* *}$ & 178.11 & 6.81 & -13.436 \\
\hline $\mathbf{4 4}$ & P5 x P9 & 47.33 & 5.37 & $24.486 * *$ & 184.67 & -4.21 & -19.926 \\
\hline $\mathbf{4 5}$ & P5 x P10 & 48.22 & -2.22 & -0.263 & 180.00 & 1.00 & -16.112 \\
\hline $\mathbf{4 6}$ & P6 x P7 & 53.33 & -4.76 & 1.077 & 182.33 & 2.74 & 0.677 \\
\hline $\mathbf{4 7}$ & P6 x P8 & 50.67 & -11.39 & 0.814 & 181.78 & 1.75 & -10.799 \\
\hline $\mathbf{4 8}$ & P6 x P9 & 51.00 & -7.89 & $22.612^{* *}$ & 186.78 & $-5.09 *+$ & -24.604 \\
\hline $\mathbf{4 9}$ & P6 x P10 & 55.33 & 4.27 & -0.722 & 185.11 & 2.16 & $144.469 * *$ \\
\hline $\mathbf{5 0}$ & P7 x P8 & 49.89 & 4.16 & 0.995 & 174.56 & -1.02 & -19.789 \\
\hline $\mathbf{5 1}$ & P7 x P9 & 50.44 & $8.37 *$ & -0.940 & 178.67 & -1.63 & -14.868 \\
\hline $\mathbf{5 2}$ & P7 x P10 & 55.22 & 0.46 & $17.302^{* *}$ & 185.56 & 0.83 & $263.605 * *$ \\
\hline $\mathbf{5 3}$ & P8 x P9 & 47.22 & -5.84 & $26.049 * *$ & 178.89 & -7.58 & -22.459 \\
\hline $\mathbf{5 4}$ & P8 x P10 & 47.22 & -0.15 & $19.542 * *$ & 179.56 & 1.81 & -4.942 \\
\hline $\mathbf{5 5}$ & P9 x P10 & 49.33 & 11.80 & $7.913 * *$ & 179.33 & $-2.06 *+$ & -24.900 \\
\hline $\mathbf{5 6}$ & NRCHB 506 & 46.44 & 2.91 & -0.825 & 189.56 & -0.27 & -22.078 \\
\hline $\mathbf{5 7}$ & DMH 1 & 43.56 & -2.02 & $8.042 * *$ & 192.11 & 2.20 & -22.928 \\
\hline
\end{tabular}

Table.3 Stability parameters for number of primary branches per plant and length of main branch [Eberhart and Russel (1966)]

\begin{tabular}{|c|c|c|c|c|c|c|c|}
\hline \multirow[t]{2}{*}{ SN } & \multirow[t]{2}{*}{ Genotype } & \multicolumn{3}{|c|}{$\begin{array}{l}\text { No. of primary branches per } \\
\text { plant }\end{array}$} & \multicolumn{3}{|c|}{ Length of main branch } \\
\hline & & $\mu$ & bi & $\mathbf{s}^{2} \mathbf{d}_{\mathrm{i}}$ & $\mu$ & bi & $\mathbf{s}^{2} \mathbf{d}_{\mathbf{i}}$ \\
\hline 1 & P1 & 6.53 & -0.91 & -0.131 & 84.36 & 1.88 & -4.379 \\
\hline 2 & P2 & 6.44 & 1.88 & 0.294 & 86.00 & 0.30 & $64.846^{* *}$ \\
\hline 3 & P3 & 6.19 & 0.10 & -0.009 & 79.56 & -4.53 & $41.533^{* * *}$ \\
\hline 4 & P4 & 7.53 & $1.93 *$ & -0.135 & 94.33 & -3.57 & $37.972^{* *}$ \\
\hline 5 & P5 & 7.27 & 0.55 & -0.133 & 98.89 & -1.56 & $69.543^{* *}$ \\
\hline 6 & P6 & 6.58 & 1.16 & $0.458 *$ & 86.67 & 1.65 & $47.807^{* * *}$ \\
\hline 7 & P7 & 6.18 & 2.41 & -0.102 & 83.56 & -8.55 & -0.951 \\
\hline 8 & P8 & 5.94 & 3.39 & -0.091 & 87.11 & 2.21 & $13.056^{*}$ \\
\hline 9 & P9 & 5.73 & 2.40 & $1.333 * *$ & 80.56 & 1.47 & -1.939 \\
\hline 10 & $\mathrm{P} 10$ & 5.76 & 0.91 & $3.351 * *$ & 93.33 & 7.36 & 4.508 \\
\hline 11 & P1 x P2 & 5.84 & -0.78 & 0.135 & 83.67 & 0.28 & 11.539 \\
\hline 12 & P1 x P3 & 5.34 & 1.10 & $0.725 *$ & 78.33 & -2.34 & $39.832 * *$ \\
\hline 13 & P1 x P4 & 6.08 & 2.96 & $0.566^{*}$ & 95.00 & 0.29 & $35.533 * *$ \\
\hline 14 & P1 x P5 & 5.86 & 0.53 & -0.133 & 94.22 & -1.26 & $30.296^{* *}$ \\
\hline 15 & P1 x P6 & 5.91 & 1.80 & -0.087 & 84.67 & -4.04 & $80.377 * *$ \\
\hline 16 & P1 x P7 & 5.79 & 0.46 & -0.105 & 85.11 & $4.38 *$ & -4.314 \\
\hline 17 & P1 x P8 & 6.29 & 0.93 & $2.029 * *$ & 81.67 & 3.51 & $18.716^{*}$ \\
\hline
\end{tabular}




\begin{tabular}{|c|c|c|c|c|c|c|c|}
\hline 18 & P1 x P9 & 6.47 & 0.28 & $1.655^{* *}$ & 77.44 & -1.47 & -1.939 \\
\hline 19 & $\mathrm{P} 1 \times \mathrm{P} 10$ & 5.69 & 0.89 & 0.211 & 89.67 & -2.63 & $92.435 * *$ \\
\hline 20 & $\mathrm{P} 2 \times \mathrm{P} 3$ & 6.40 & 1.35 & -0.074 & 85.89 & 2.43 & $79.753 * *$ \\
\hline 21 & $\mathrm{P} 2 \times \mathrm{P} 4$ & 6.66 & 3.44 & -0.095 & 86.78 & -2.17 & $49.224 * *$ \\
\hline 22 & P2 x P5 & 6.47 & 1.90 & -0.039 & 95.00 & 0.35 & $29.896 * *$ \\
\hline 23 & P2 x P6 & 6.16 & $2.19 *$ & -0.131 & 84.33 & 2.96 & -4.416 \\
\hline 24 & P2 x P7 & 5.70 & 0.48 & -0.113 & 85.33 & 1.25 & $15.372 *$ \\
\hline 25 & $\mathrm{P} 2 \times \mathrm{P} 8$ & 5.86 & 1.03 & $0.746^{*}$ & 82.89 & 2.26 & -1.379 \\
\hline 26 & $\mathrm{P} 2 \times \mathrm{P} 9$ & 5.94 & -2.03 & 0.053 & 81.78 & -2.36 & 9.759 \\
\hline 27 & $\mathrm{P} 2 \times \mathrm{P} 10$ & 5.93 & -2.20 & $1.912 * *$ & 93.33 & -5.29 & -3.147 \\
\hline 28 & P3 x P4 & 7.00 & 0.85 & 0.258 & 89.67 & 4.34 & $20.922 *$ \\
\hline 29 & P3 x P5 & 6.68 & 2.16 & 0.287 & 91.78 & 2.33 & 4.218 \\
\hline 30 & P3 x P6 & 6.53 & -0.49 & -0.124 & 87.00 & -1.11 & 7.978 \\
\hline 31 & P3 x P7 & 6.24 & 1.80 & -0.128 & 79.00 & 5.22 & -4.020 \\
\hline 32 & P3 x P8 & 6.54 & 0.52 & -0.087 & 88.11 & 6.56 & -3.322 \\
\hline 33 & P3 x P9 & 5.66 & 0.07 & $3.530 * *$ & 81.67 & 1.18 & 11.461 \\
\hline 34 & P3 x P10 & 6.56 & 1.40 & -0.022 & 80.89 & 5.19 & $20.925^{*}$ \\
\hline 35 & P4 x P5 & 6.33 & 2.10 & $0.527 *$ & 99.78 & $6.76^{*}+$ & -4.449 \\
\hline 36 & P4 x P6 & 5.91 & 2.63 & 0.215 & 99.78 & -2.45 & -3.529 \\
\hline 37 & P4 x P7 & 6.27 & 1.78 & $1.208 * *$ & 85.89 & 1.98 & 0.455 \\
\hline 38 & P4 x P8 & 6.56 & 3.34 & -0.028 & 93.22 & 2.43 & 6.479 \\
\hline 39 & P4 x P9 & 6.79 & 0.23 & -0.115 & 87.22 & 3.45 & $16.296 *$ \\
\hline 40 & P4 x P10 & 6.06 & 1.38 & 0.101 & 90.00 & 6.21 & $44.624 * *$ \\
\hline 41 & P5 x P6 & 6.61 & 2.21 & $0.783 * *$ & 92.56 & 0.04 & $47.316 * *$ \\
\hline 42 & P5 x P7 & 6.28 & 2.69 & -0.108 & 89.78 & 6.88 & -3.631 \\
\hline 43 & P5 x P8 & 5.22 & 2.15 & $2.712 * *$ & 85.78 & 7.05 & $31.986 * *$ \\
\hline 44 & P5 x P9 & 6.00 & 0.08 & -0.131 & 90.89 & -5.39 & 4.103 \\
\hline 45 & P5 x P10 & 6.17 & 2.04 & 0.320 & 86.00 & 2.30 & 3.322 \\
\hline 46 & P6 x P7 & 5.87 & 1.05 & 0.029 & 93.11 & -0.89 & $74.479 * *$ \\
\hline 47 & P6 x P8 & 6.07 & 0.78 & -0.068 & 91.67 & 5.29 & -3.146 \\
\hline 48 & P6 x P9 & 6.23 & 1.16 & -0.017 & 92.56 & -3.43 & $62.119 * *$ \\
\hline 49 & P6 x P10 & 6.19 & -2.23 & $1.847 * *$ & 88.78 & 1.96 & $29.498 * *$ \\
\hline 50 & P7 x P8 & 5.58 & -1.06 & -0.126 & 83.11 & -2.33 & 0.476 \\
\hline 51 & P7 x P9 & 5.60 & -0.01 & 0.212 & 90.78 & 4.85 & 9.176 \\
\hline 52 & $\mathrm{P} 7 \times \mathrm{P} 10$ & 6.18 & 0.26 & -0.047 & 90.11 & 4.13 & -3.926 \\
\hline 53 & P8 x P9 & 5.68 & 0.47 & $1.119 * *$ & 90.00 & -1.89 & $18.154^{*}$ \\
\hline 54 & P8 x P10 & 5.72 & 1.06 & $0.480^{*}$ & 86.22 & 1.73 & -0.839 \\
\hline 55 & $\mathrm{P} 9 \times \mathrm{P} 10$ & 5.61 & -0.27 & -0.118 & 87.11 & -0.06 & -3.576 \\
\hline 56 & NRCHB 506 & 6.77 & $0.37 *+$ & -0.136 & 94.56 & -2.72 & 8.219 \\
\hline 57 & DMH 1 & 6.39 & 0.36 & -0.112 & 92.33 & $4.57 *$ & -4.360 \\
\hline
\end{tabular}


Table.4 Stability parameters for seed yield per plant and 1000-seed weight [Eberhart and Russel (1966)]

\begin{tabular}{|c|c|c|c|c|c|c|c|}
\hline \multirow[t]{2}{*}{$\mathbf{S N}$} & \multirow[t]{2}{*}{ Genotype } & \multicolumn{3}{|c|}{ Seed yield per plant } & \multicolumn{3}{|c|}{ 1000-Seed weight } \\
\hline & & $\mu$ & bi & $s^{2} d_{i}$ & $\mu$ & bi & $s^{2} d_{i}$ \\
\hline 1 & $\mathrm{P} 1$ & 30.61 & 6.29 & -2.794 & 4.64 & 1.23 & 0.054 \\
\hline 2 & $\mathrm{P} 2$ & 34.32 & 1.94 & -1.590 & 5.69 & $-6.14+$ & -0.034 \\
\hline 3 & P3 & 36.39 & -0.65 & 5.239 & 5.27 & 2.68 & $0.145^{*}$ \\
\hline 4 & $\mathrm{P} 4$ & 39.44 & 2.03 & $11.995 *$ & 6.13 & $-2.00 *+$ & -0.035 \\
\hline 5 & P5 & 32.81 & 7.60 & $19.540 * *$ & 5.35 & -3.36 & -0.025 \\
\hline 6 & P6 & 29.03 & -3.17 & 4.089 & 4.51 & 2.37 & -0.032 \\
\hline 7 & P7 & 28.10 & -0.00 & $32.450 * *$ & 4.51 & 3.38 & -0.030 \\
\hline 8 & P8 & 29.86 & 1.11 & 7.964 & 4.71 & 6.41 & $0.292 * *$ \\
\hline 9 & P9 & 32.49 & 4.05 & $29.194 * *$ & 4.46 & -3.40 & 0.067 \\
\hline 10 & P10 & 25.39 & -3.55 & 1.752 & 5.33 & -2.05 & 0.039 \\
\hline 11 & $\mathrm{P} 1 \times \mathrm{P} 2$ & 31.97 & -6.36 & 3.093 & 4.57 & -1.20 & $0.217 * *$ \\
\hline 12 & P1 x P3 & 32.70 & 1.89 & $11.930 *$ & 4.74 & -8.12 & $0.539 * *$ \\
\hline 13 & P1 x P4 & 36.88 & 0.26 & 6.243 & 5.32 & -2.66 & 0.078 \\
\hline 14 & P1 x P5 & 31.44 & 0.57 & $20.802 * *$ & 4.79 & 2.00 & $1.083^{* *}$ \\
\hline 15 & P1 x P6 & 31.94 & 5.81 & $16.506^{* *}$ & 3.89 & 1.47 & -0.014 \\
\hline 16 & P1 x P7 & 31.02 & 1.44 & $8.862 *$ & 4.10 & 9.49 & $0.366^{* *}$ \\
\hline 17 & P1 x P8 & 26.53 & -0.62 & -2.035 & 4.14 & 2.24 & $0.121 *$ \\
\hline 18 & P1 x P9 & 32.51 & -1.10 & -1.747 & 4.89 & 0.47 & $0.128 *$ \\
\hline 19 & P1 x P10 & 35.58 & 2.85 & -1.369 & 4.96 & 10.23 & $0.236 * *$ \\
\hline 20 & P2 x P3 & 33.89 & -3.42 & 6.506 & 4.82 & 6.71 & $0.334 * *$ \\
\hline 21 & $\mathrm{P} 2 \times \mathrm{P} 4$ & 39.99 & $-11.41 * *++$ & -2.893 & 5.36 & -3.89 & $1.174 * *$ \\
\hline 22 & P2 x P5 & 37.87 & -0.64 & $45.529 * *$ & 4.84 & 2.71 & $0.435 * *$ \\
\hline 23 & P2 x P6 & 27.47 & 0.77 & 5.966 & 4.35 & 1.34 & $0.584 * *$ \\
\hline 24 & P2 x P7 & 34.10 & $11.74 *+$ & -2.866 & 4.89 & 1.43 & $0.248 * *$ \\
\hline 25 & P2 x P8 & 36.62 & 0.67 & $9.712 *$ & 4.77 & 9.92 & -0.022 \\
\hline 26 & $\mathrm{P} 2 \times \mathrm{P} 9$ & 35.99 & 14.74 & -1.428 & 4.05 & -2.80 & 0.020 \\
\hline 27 & $\mathrm{P} 2 \times \mathrm{P} 10$ & 38.74 & 3.46 & 5.552 & 4.88 & 5.17 & $0.185^{*}$ \\
\hline 28 & P3 x P4 & 42.78 & -1.77 & -1.375 & 4.87 & -2.68 & -0.020 \\
\hline 29 & P3 x P5 & 37.46 & 0.11 & -2.718 & 4.94 & 0.53 & 0.088 \\
\hline 30 & P3 x P6 & 33.61 & -4.00 & $14.581 *$ & 4.69 & -0.01 & $0.253 * *$ \\
\hline 31 & P3 x P7 & 34.76 & 11.65 & $15.939 *$ & 5.06 & -1.23 & 0.083 \\
\hline 32 & P3 x P8 & 26.65 & 0.50 & $9.143 *$ & 4.64 & 12.14 & $0.124^{*}$ \\
\hline 33 & P3 x P9 & 27.39 & -3.86 & 6.372 & 4.03 & -3.13 & $1.088 * *$ \\
\hline 34 & P3 x P10 & 35.34 & -9.81 & -0.703 & 4.58 & 0.43 & 0.005 \\
\hline 35 & $\mathrm{P} 4 \times \mathrm{P} 5$ & 38.32 & -9.51 & $23.515^{* *}$ & 4.77 & -8.14 & 0.006 \\
\hline 36 & P4 x P6 & 34.21 & 4.36 & $96.860 * *$ & 4.83 & -7.71 & $0.218 * *$ \\
\hline 37 & P4 x P7 & 33.36 & -3.02 & 4.331 & 5.32 & -1.86 & 0.039 \\
\hline 38 & P4 x P8 & 36.01 & -10.13 & $28.415^{* *}$ & 5.08 & $7.19 *+$ & -0.035 \\
\hline
\end{tabular}




\begin{tabular}{|l|l|r|r|r|r|r|c|}
\hline $\mathbf{3 9}$ & P4 x P9 & 43.64 & -0.87 & 0.672 & 5.11 & 1.65 & $0.453^{* *}$ \\
\hline $\mathbf{4 0}$ & P4 x P10 & 41.63 & 1.30 & 7.484 & 4.58 & -7.35 & -0.013 \\
\hline $\mathbf{4 1}$ & P5 x P6 & 32.34 & -9.26 & $10.760^{*}$ & 4.88 & 4.45 & 0.014 \\
\hline $\mathbf{4 2}$ & P5 x P7 & 28.03 & -0.86 & 1.336 & 4.45 & 2.67 & -0.004 \\
\hline $\mathbf{4 3}$ & P5 x P8 & 26.15 & 10.57 & $10.339^{*}$ & 4.69 & 5.98 & -0.017 \\
\hline $\mathbf{4 4}$ & P5 x P9 & 27.67 & 6.10 & -1.168 & 4.16 & 4.33 & $0.115^{*}$ \\
\hline $\mathbf{4 5}$ & P5 x P10 & 30.77 & -9.75 & $22.527^{* *}$ & 3.86 & -3.61 & $0.129^{*}$ \\
\hline $\mathbf{4 6}$ & P6 x P7 & 28.05 & 4.37 & 3.911 & 4.10 & -2.83 & 0.042 \\
\hline $\mathbf{4 7}$ & P6 x P8 & 28.88 & 7.00 & -1.352 & 4.43 & $-4.41^{* *++}$ & -0.035 \\
\hline $\mathbf{4 8}$ & P6 x P9 & 34.34 & -0.20 & 5.518 & 4.94 & 4.62 & $0.118^{*}$ \\
\hline $\mathbf{4 9}$ & P6 x P10 & 26.94 & 8.89 & $14.748^{*}$ & 4.42 & -1.30 & $0.883^{* *}$ \\
\hline $\mathbf{5 0}$ & P7 x P8 & 33.43 & 11.21 & $19.341^{* *}$ & 3.95 & 13.33 & 0.055 \\
\hline $\mathbf{5 1}$ & P7 x P9 & 35.13 & 3.52 & $9.526^{*}$ & 4.25 & 3.69 & $0.155^{*}$ \\
\hline $\mathbf{5 2}$ & P7 x P10 & 36.55 & 3.29 & 5.078 & 4.18 & 9.82 & $0.322^{* *}$ \\
\hline $\mathbf{5 3}$ & P8 x P9 & 27.81 & 11.73 & 0.500 & 4.17 & 2.01 & $0.131^{*}$ \\
\hline $\mathbf{5 4}$ & P8 x P10 & 25.10 & 7.06 & 4.606 & 4.11 & -6.53 & $0.286^{* *}$ \\
\hline $\mathbf{5 5}$ & P9 x P10 & 33.22 & -3.84 & $8.432^{*}$ & 4.46 & -0.77 & 0.033 \\
\hline $\mathbf{5 6}$ & NRCHB & 40.40 & -0.36 & 0.272 & 4.94 & 7.50 & 0.079 \\
\hline & 506 & & & & & & \\
\hline $\mathbf{5 7}$ & DMH 1 & 39.03 & -3.72 & 0.491 & 4.91 & -5.36 & $0.117^{*}$ \\
\hline
\end{tabular}

Table.5 Stability parameters for harvest index and protein content [Eberhart and Russel (1966)]

\begin{tabular}{|l|l|l|r|r|r|r|r|}
\hline SN & Genotype & \multicolumn{3}{|c|}{ Harvest index } & \multicolumn{3}{|c|}{ Protein content } \\
\hline & & $\boldsymbol{\mu}$ & $\mathbf{b i}$ & $\mathbf{s}^{2} \mathbf{d}_{\mathbf{i}}$ & $\boldsymbol{\mu}$ & $\mathbf{b i}$ & $\mathbf{s}^{\mathbf{2}} \mathbf{d}_{\mathbf{i}}$ \\
\hline $\mathbf{1}$ & P1 & 39.36 & 3.49 & -6.038 & 30.30 & -0.47 & -0.066 \\
\hline $\mathbf{2}$ & P2 & 44.13 & 2.54 & 6.263 & 31.07 & 4.63 & -0.364 \\
\hline $\mathbf{3}$ & P3 & 48.63 & -1.44 & -2.157 & 30.48 & 5.78 & -0.361 \\
\hline $\mathbf{4}$ & P4 & 46.18 & 2.24 & 2.435 & 32.54 & -0.07 & -0.289 \\
\hline $\mathbf{5}$ & P5 & 40.04 & 10.39 & 16.179 & 30.59 & -6.43 & 0.688 \\
\hline $\mathbf{6}$ & P6 & 38.80 & -1.51 & -5.058 & 30.79 & -5.00 & -0.179 \\
\hline $\mathbf{7}$ & P7 & 38.23 & 0.86 & $45.444^{* *}$ & 29.94 & -5.83 & 0.244 \\
\hline $\mathbf{8}$ & P8 & 35.48 & 6.12 & 8.376 & 29.51 & -4.27 & -0.109 \\
\hline $\mathbf{9}$ & P9 & 46.14 & 6.14 & $175.459^{* *}$ & 30.26 & 0.58 & -0.213 \\
\hline $\mathbf{1 0}$ & P10 & 33.59 & -3.00 & -1.037 & 30.70 & 0.51 & -0.395 \\
\hline $\mathbf{1 1}$ & P1 x P2 & 43.45 & -1.82 & -2.340 & 29.81 & -5.82 & 0.140 \\
\hline $\mathbf{1 2}$ & P1 x P3 & 40.47 & -4.29 & $321.555^{* *}$ & 30.45 & 1.93 & 0.087 \\
\hline $\mathbf{1 3}$ & P1 x P4 & 54.28 & 4.43 & $34.161^{*}$ & 30.95 & 6.05 & -0.173 \\
\hline $\mathbf{1 4}$ & P1 x P5 & 39.57 & 3.03 & 17.220 & 30.40 & -5.92 & -0.179 \\
\hline $\mathbf{1 5}$ & P1 x P6 & 38.84 & 1.68 & $59.018^{* *}$ & 30.20 & -2.09 & -0.288 \\
\hline $\mathbf{1 6}$ & P1 x P7 & 39.36 & -3.46 & -6.934 & 29.13 & -3.30 & 0.642 \\
\hline & & & & & & & \\
\hline
\end{tabular}




\begin{tabular}{|c|c|c|c|c|c|c|c|}
\hline 17 & P1 x P8 & 37.10 & 2.56 & $114.907 * *$ & 28.03 & -1.59 & -0.295 \\
\hline 18 & P1 x P9 & 38.80 & -1.33 & $85.710^{* *}$ & 29.74 & -2.07 & 0.214 \\
\hline 19 & P1 x P10 & 45.94 & -6.03 & $350.689^{* *}$ & 27.00 & 4.09 & -0.357 \\
\hline 20 & $\mathrm{P} 2 \times \mathrm{P} 3$ & 41.11 & -2.63 & $27.050^{*}$ & 30.54 & $-0.50+$ & -0.395 \\
\hline 21 & $\mathrm{P} 2 \times \mathrm{P} 4$ & 45.51 & -2.47 & $29.617^{*}$ & 27.12 & 5.50 & -0.192 \\
\hline 22 & $\mathrm{P} 2 \times \mathrm{P} 5$ & 52.09 & 1.29 & $67.587 * *$ & 31.32 & -0.07 & -0.046 \\
\hline 23 & P2 x P6 & 34.14 & 0.15 & 11.620 & 26.50 & -0.64 & -0.388 \\
\hline 24 & $\mathrm{P} 2 \times \mathrm{P} 7$ & 40.79 & 5.71 & -3.408 & 27.00 & 7.28 & 0.465 \\
\hline 25 & $\mathrm{P} 2 \times \mathrm{P} 8$ & 49.47 & -0.36 & -4.931 & 26.10 & -0.50 & -0.185 \\
\hline 26 & $\mathrm{P} 2 \times \mathrm{P} 9$ & 50.82 & 9.77 & 6.958 & 30.22 & -4.75 & -0.277 \\
\hline 27 & $\mathrm{P} 2 \times \mathrm{P} 10$ & 42.65 & -0.63 & $41.232 * *$ & 30.31 & 0.92 & 0.140 \\
\hline 28 & P3 x P4 & 42.90 & -1.47 & -5.957 & 31.42 & 9.28 & -0.365 \\
\hline 29 & P3 x P5 & 39.56 & 0.06 & -4.521 & 30.14 & 10.12 & -0.292 \\
\hline 30 & P3 x P6 & 44.37 & -1.43 & 4.616 & 30.11 & 5.94 & $2.846^{* *}$ \\
\hline 31 & P3 x P7 & 41.06 & 3.44 & -1.736 & 30.16 & 0.49 & 0.351 \\
\hline 32 & P3 x P8 & 36.61 & -1.18 & -7.176 & 27.09 & 0.99 & -0.368 \\
\hline 33 & P3 x P9 & 41.76 & -0.77 & -4.524 & 30.49 & 4.84 & 0.883 \\
\hline 34 & $\mathrm{P} 3 \times \mathrm{P} 10$ & 40.54 & -2.21 & -2.142 & 30.76 & -2.96 & -0.391 \\
\hline 35 & P4 x P5 & 40.31 & $-0.86+$ & -7.220 & 29.56 & 8.79 & -0.120 \\
\hline 36 & P4 x P6 & 38.95 & 2.91 & $83.391 * *$ & 29.91 & 9.85 & 0.279 \\
\hline 37 & P4 x P7 & 42.16 & -3.14 & -5.738 & 28.98 & 9.56 & -0.088 \\
\hline 38 & P4 x P8 & 43.86 & -4.65 & $245.289 * *$ & 28.52 & 10.31 & 0.112 \\
\hline 39 & P4 x P9 & 47.08 & 4.79 & -6.475 & 30.79 & 1.49 & -0.212 \\
\hline 40 & P4 x P10 & 45.56 & 1.26 & 7.526 & 30.40 & -1.31 & 0.433 \\
\hline 41 & P5 x P6 & 42.35 & 5.48 & 5.346 & 27.17 & -2.83 & -0.334 \\
\hline 42 & P5 x P7 & 38.88 & 3.06 & $76.672 * *$ & 30.05 & 0.58 & -0.381 \\
\hline 43 & P5 x P8 & 43.31 & 7.66 & $123.345^{* *}$ & 28.70 & -5.66 & 0.342 \\
\hline 44 & P5 x P9 & 41.68 & 0.80 & 1.605 & 27.17 & 8.97 & -0.091 \\
\hline 45 & P5 x P10 & 39.01 & -3.57 & $101.567 * *$ & 28.55 & 4.39 & 0.707 \\
\hline 46 & P6 x P7 & 40.64 & 0.65 & $37.269 *$ & 25.71 & -2.02 & -0.185 \\
\hline 47 & P6 x P8 & 36.71 & 1.50 & $91.555^{* *}$ & 27.72 & -7.43 & -0.227 \\
\hline 48 & P6 x P9 & 43.48 & -5.30 & $64.948 * *$ & 29.23 & -3.96 & -0.230 \\
\hline 49 & P6 x P10 & 33.42 & 4.73 & $44.586^{* *}$ & 29.46 & 5.70 & -0.176 \\
\hline 50 & P7 x P8 & 36.07 & 4.46 & 19.360 & 27.50 & -4.78 & -0.300 \\
\hline 51 & P7 x P9 & 40.07 & -0.09 & -5.661 & 27.15 & 4.61 & -0.276 \\
\hline 52 & $\mathrm{P} 7 \times \mathrm{P} 10$ & 44.31 & 1.05 & $90.851 * *$ & 28.64 & 1.06 & -0.394 \\
\hline 53 & P8 x P9 & 40.58 & 3.02 & 14.165 & 29.96 & 2.89 & -0.279 \\
\hline 54 & P8 $x$ P10 & 40.58 & 2.86 & 11.934 & 27.09 & 2.69 & -0.214 \\
\hline 55 & P9 $x$ P10 & 45.33 & -0.81 & $265.620 * *$ & 26.46 & -7.30 & -0.040 \\
\hline 56 & $\begin{array}{l}\text { NRCHB } \\
506\end{array}$ & 47.52 & 1.67 & -2.935 & 28.57 & -7.72 & $5.472 * *$ \\
\hline 57 & DMH 1 & 48.42 & 1.65 & 0.970 & 29.70 & 12.41 & $2.587 * *$ \\
\hline
\end{tabular}


While among crosses, cross Bio-902 $\times$ DRMR-IJ-31 showed higher mean (5.08 g) than mean of crosses (4.60 g), non-significant deviation from regression and regression coefficient more than unity so that it showed suitability for favorable environment.

None of the parent and none of the cross showed significant regression coefficient.For protein content, only one cross Pusa Vijay $\times$ PM-30showed higher mean $(30.54 \%)$ for protein content over the mean of crosses $(28.97 \%)$. It showed non-significant deviation from regression with regression coefficient less than one and showed its suitability for unfavourable environment.

The similar results for seed yield and its components and quality traits were found by Yadava et al., (2010), Mahto and Haider (2012), Gazal et al., (2013), Sagolsem et al., (2013) and Sah et al., (2015).

\section{References}

Baker, R. J. H. (1969). Genotype x environment interaction in yield of wheat. J. Pl. Sci., 49: 743-751.

Bradshaw, A.D. (1965). Evolutionary significance of plasticity in plants. Advances in Genet., 13: 115-155.

Eberhart, S.A. and Russell, W.A. (1966). Stability parameters for comparing varieties. Crop Sci., 16:36-40.

Gazal, A., Dar, Z. A., Zafar, G. and Habib, M.
(2013). Stability analysis for yield and its contributing traits in Brown sarson (Brassica rapa L.) under Kashmir conditions in India. $J$ of Oilseed Brassica, 4(1): 33-38.

Mahto, J. L. and Haider, Z. A. (2012). Combining ability and stability study for yield and oil in Indian mustard in Alfisols of Jharkhand. Cruciferae Newsletter., 31: 63-67.

Sagolsem, D., Singh, N. N., Devi, Th. R., Shabir H. Wani, S. H., Haribhushan, A., Singh, N. G. and Laishram, J. M. (2013). Genotype X environment interaction in Indian mustard (Brassica juncea L. Czern and coss) under Manipur valley conditions. Indian $J$. Genet., 73(3): 332-334.

Sah, R. P., Kumar, A., Ghosh, J. and Prasad, K. (2015). Stability study in Indian mustard (Brassica juncea L.). Journal of Hill Agriculture., 6(1): 40-44.

Sprague, G.F. and Federer, W.T. (1951). A comparison of variance components in corn yield trials II error, year x variety, variety $\mathrm{x}$ location and variety components. Agron. J., 43: 535-541.

Yadava, D. K., Giri, S. C., Vasudev, S., Yadav, A. K., Dass, B., Raje, R. S., Vignesh, M., Singh, R., Mohapatra, T. and Prabhu, K. V. (2010). Stability analysis in Indian mustard (Brassica juncea) varieties. Indian J of Agric. Sci., 80 (9): 761-765.

\section{How to cite this article:}

Neelam Shekhawat, Hemlata Sharma and Bheeru Lal Meena. 2020. Assessment of Genotype $\times$ Environment Interaction and Stability Analysis in Indian Mustard (Brassica Juncea L.). Int.J.Curr.Microbiol.App.Sci. 9(04): 1766-1776. doi: https://doi.org/10.20546/ijcmas.2020.904.207 